\title{
Formation and evolution of massive elliptical galaxies in clusters: a consistent picture from optical and X-ray properties
}

\author{
Antonio Pipino* \\ Dipartimento di Astronomia, Università di Trieste, Italy \\ E-mail: antonio@ts.astro.it \\ Francesca Matteucci \\ Dipartimento di Astronomia, Università di Trieste, Italy \\ E-mail: matteucciets.astro.it

\section{Daisuke Kawata} \\ Centre for Astrophysics and Supercomputing, Swinburne University of Technology, Australia \\ E-mail: dkawata@astro.swin.edu.au

\section{Brad K. Gibson} \\ Centre for Astrophysics and Supercomputing, Swinburne University of Technology, Australia \\ E-mail: bgibsoneastro.swin.edu.au
}

We present preliminary results from a new chemical evolution model meant to be a first step in the self-consistent study of both optical and X-ray properties of elliptical galaxies in cluster of galaxies. Detailed cooling and heating processes in the interstellar medium are taken into account using a mono-phase one-zone treatment which allows a more reliable modelling of the galactic wind regime with respect to previous work. The model successfully reproduces simultaneously the mass-metallicity, colour-magnitude, the $L_{X}-L_{B}$ and the $L_{X}-T$ relations, as well as the observed trend of the $\left[\langle\mathrm{Mg} / \mathrm{Fe}\rangle_{*}\right]$ ratio as a function of $\sigma$, by assuming that the gas infall and star formation timescales are shorter for brighter objects. We found that a late secondary accretion of gas from the environment might play a role in driving the $L_{X}-L_{B}$ and $L_{X}-T$ relations and can explain their large observational scatter. The iron discrepancy, namely the too high predicted iron abundance in X-ray haloes of ellipticals compared to observations, still persists. On the other hand, we predict $[\mathrm{O} / \mathrm{Fe}]$ in the ISM which is in good agreement with the most recent observations. New predictions for the amounts of iron, oxygen and energy ejected into the intracluster medium are presented and we conclude that Type Ia supernovae play a fundamental role in the ICM enrichment. SNe Ia activity, in fact, may power a galactic wind lasting for a considerable amount of the galactic lifetime, even in the case for which the efficiency of energy transfer into the ISM per SN Ia event is less than unity.

Baryons in Dark Matter Halos

5-9 October 2004

Novigrad, Croatia

${ }^{*}$ Speaker. 


\section{Introduction}

The most direct evidence for the presence of a non-negligible interstellar medium (ISM) in elliptical galaxies comes from X-ray observations. The presence of $\sim 10^{8}-10^{10} M_{\odot}$ of gas in a hot $\left(\sim 10^{7} \mathrm{~K}\right) \mathrm{X}$-ray emitting $\left(L_{X} \sim 10^{39}-10^{42} \mathrm{erg} \mathrm{s}^{-1}\right)$ phase, was first discovered by the Einstein satellite for ellipticals with a wide range of optical luminosities. A correlation between X-ray $\left(L_{X}\right)$ and optical $\left(L_{B}\right)$ luminosities is observed with roughly $L_{X} \propto L_{B}^{2}$ (e.g. O'Sullivan et al. 2001), although the scatter is quite large (see Mathews \& Brighenti 2003 for a recent review on this topic). Such a trend can be explained by the fact that optically brighter galaxies are also more gravitationally bound (Ciotti et al. 1991). X-ray spectra also carry information pertaining to the chemical composition of the interstellar gas. The ASCA satellite provided the first reliable measure of the iron abundance in the hot ISM of ellipticals (e.g. Matsumoto et al. 1997). These observations led to the so-called iron discrepancy (Arimoto et al. 1997), namely the too high predicted iron abundance in X-ray haloes of ellipticals compared to observations, being oversolar the former and well below the solar value for the $\mathrm{Fe}$ abundance $\left(\mathrm{Fe}_{\odot}\right)$ the latter. New telescopes, now provide us with more robust $\mathrm{Fe}$ abundances, which are $\sim 1-2 \mathrm{Fe}_{\odot}$. The aim of this work is to improve the detailed photo-chemical evolution code of Pipino \& Matteucci (2004, PM04) with a new treatment of the hot ISM, in order to present, for the first time, a model able to predict in a self-consistent manner the properties of both stellar (i.e. optical) and hot gas (i.e. X-ray) components of the flux emitted by elliptical galaxies.

\section{The model}

The adopted chemical evolution model is based on that presented by PM04. In this particular case, however, we consider our model galaxies as a single zone extending out to 10 effective radii, with instantaneous mixing of gas. Moreover we take explicitly into account a possible mass flow due to the galactic wind and a possible secondary episode of gas accretion in order to model late time gas accretion and/or interactions with the environment. The chemical code features a new self-consistent energetic treatment which supersedes the previous one adopted by PM04 (we refer the reader to Pipino et al., 2005, for a detailed description). In the case of secondary accretion, we take into account that the infalling gas has a mean energy assumed to be equal to the virial temperature, therefore influencing the final ISM temperature. Detailed cooling (Kawata \& Gibson, 2003a) and heating of the ISM are taken into account. Particular care is dedicated to a detailed calculations of Type Ia and II SN rates. In this paper we define the time when the galactic wind onsets $\left(t_{\mathrm{gw}}\right)$ when the gas thermal energy becomes greater than the binding energy. For every timestep in which the thermal energy is greater than the binding energy, we set the energy loss in the wind as the value given by $\Delta E=E_{\mathrm{th}}-E_{\mathrm{Bin}}$, whereas in previous works (Pipino et al., 2002, P02; PM04) all the thermal energy was allowed to escape. The ejected mass is given by $\frac{\Delta M}{M_{\mathrm{gas}}}=\frac{\Delta E}{E_{\mathrm{th}}}$. The SN efficiency before $t_{\mathrm{gw}}$ is assumed to be $10 \%$. For the secondary accretion, we assume that the model galaxy accretes a mass $M_{\text {acc }}$ of primordial composition gas in a uniform way all along its lifetime. In order to predict reliable estimates for $L_{X}$ and $T$ we simulated observations of our model galaxies and applied the procedure described by Kawata \& Gibson (2003b), for XSPEC ver.11.1.0 package.

$091 / 2$ 
Table 1: Input parameters and model predictions

\begin{tabular}{|c|c|c|c|c|c|c|c|c|c|c|c|c|}
\hline Model & $\begin{array}{l}M_{\text {lum }} \\
\left(M_{\odot}\right) \\
\end{array}$ & $\begin{array}{l}R_{\text {eff }} \\
(\mathrm{kpc})\end{array}$ & $\begin{array}{l}v \\
\left(\mathrm{Gyr}^{-1}\right)\end{array}$ & $\begin{array}{l}\tau \\
(\mathrm{Gyr})\end{array}$ & $\begin{array}{l}t_{\mathrm{gw}} \\
(\mathrm{Gyr})\end{array}$ & $\frac{M_{\text {acc }}}{M_{\text {lum }}}$ & $\begin{array}{l}\begin{array}{l}\text { w. stop } \\
\text { (Gyr) }\end{array} \\
\end{array}$ & $\frac{M_{\text {gas }}}{M_{\text {lum }}}$ & $\overline{[\mathrm{Fe} / \mathrm{H}]}$ & $\overline{[\mathrm{O} / \mathrm{Fe}]}$ & $\begin{array}{l}L_{X} \\
\left(10^{41} \mathrm{erg} / \mathrm{s}\right)\end{array}$ & $\begin{array}{l}\mathrm{kT}_{\mathrm{X}} \\
(\mathrm{keV})\end{array}$ \\
\hline L0 & $10^{11}$ & 3 & 15 & 0.3 & 0.82 & 0 & never & 0.00014 & 0.85 & -0.56 & $9.3 \cdot 10^{-3}$ & 1.2 \\
\hline La1 & $10^{11}$ & 3 & 15 & 0.3 & 0.82 & 0.01 & 9.8 & 0.014 & 0.85 & -0.68 & 2 & 0.5 \\
\hline La10 & $10^{11}$ & 3 & 15 & 0.3 & 0.82 & 0.1 & 6.34 & 0.15 & 0.92 & -1.05 & - & 0.001 \\
\hline H0 & $10^{12}$ & 10 & 25 & 0.2 & 0.46 & 0 & never & 0.003 & 1.23 & -0.87 & 16.7 & 1.7 \\
\hline Ha1 & $10^{12}$ & 10 & 25 & 0.2 & 0.46 & 0.01 & 6.0 & 0.05 & 1.3 & -1.04 & 183 & 1 \\
\hline Ha10 & $10^{12}$ & 10 & 25 & 0.2 & 0.46 & 0.1 & 1.94 & 0.17 & 1.60 & -1.17 & - & 0.001 \\
\hline
\end{tabular}

\section{Results and conclusions}

A summary of the input parameters as well as results are shown in Table 1, where model name (Col. 1), mass (Col. 2), effective radius (Col. 3), SF efficiency (Col. 4), infall timescale (Col. 5), accreted mass in the secondary episode (Col. 7) as well as the times at which the galactic wind starts and stops (Col. 8 and 8, respectively) are given. For each model we show how crucial is the role played by secondary accretion in determining the final hot gas mass (Col. 9), ISM chemical abundances (Cols. 10 and 11), X-ray luminosity (Col. 12) and, finally, ISM temperature (Col. 13).

Our new models confirm the previous finding of PM04, namely that SF and infall timescales decreasing with galactic mass are needed to explain the optical properties of elliptical galaxies (e.g. the Color-Magnitude relation, see Fig. 1, left panel). At the same time we reproduce the $L_{X}-L_{B}$ relation in the ISM of bright ellipticals. In Fig. 1 (right panel) we show our predictions for model La1 and Ha1 (filled triangles), compared with the mean relation derived from the large sample of O'Sullivan et al. (2001, solid line). Our points lie well within the observed region (a measure of the scatter is given by the dotted lines), although the predicted slope seems to be slightly flatter with respect to the fitted relations.

An interesting result is that the scatter in the observed $L_{B}-L_{X}$ relation can be totally explained either by small differences in the accretion history or variations in density. In order to show this, we plot in Fig. 1 models with $M_{\text {acc }}=0.005 M_{\text {lum }}$ and $M_{\text {acc }}=0.007 M_{\text {lum }}$ (filled squares and stars, respectively). The difference in the predicted $L_{X}$ at a given $L_{B}$ for these two models and the fiducial case spans the same region that is between the fit to the observed data (solid line) and the $1 \sigma$ boundary (dotted line) in the case of the high mass model. For the low mass one, instead, the range in the predicted values covers a much larger region. The secondary mild inflow seems to play a non-negligible role in governing the late-time behaviour of the ISM, in particular in driving $L_{X}-L_{B}$ and $L_{X}-T_{X}$ relations, whereas its effects on the colours and the line-strength indices are negligible. The iron discrepancy between model predictions and observations seems still to persist, probably indicating the presence of other mechanisms acting at galactic scales. We suggest that the mixing of gas driven by AGNs can preserve the gas mass (and thus the X-ray luminosity) while diluting the iron abundance. The new energetic formalism implemented in the chemical evolution code allows us to follow in a more detailed way the evolution of mass and energy flow into the ICM with respect to previous work (e.g. P02). Nevertheless, our preliminary results show that the predicted amount of $\mathrm{Fe},[\alpha / \mathrm{Fe}]$ ratios as well as the energy injected into the ICM are very similar to P02 results, with no evolution of ICM abundances out to redshift 1 (Tozzi et al., 2003). Therefore, we confirm that SNe Ia are fundamental in providing energy and iron to the ICM.

$091 / 3$ 

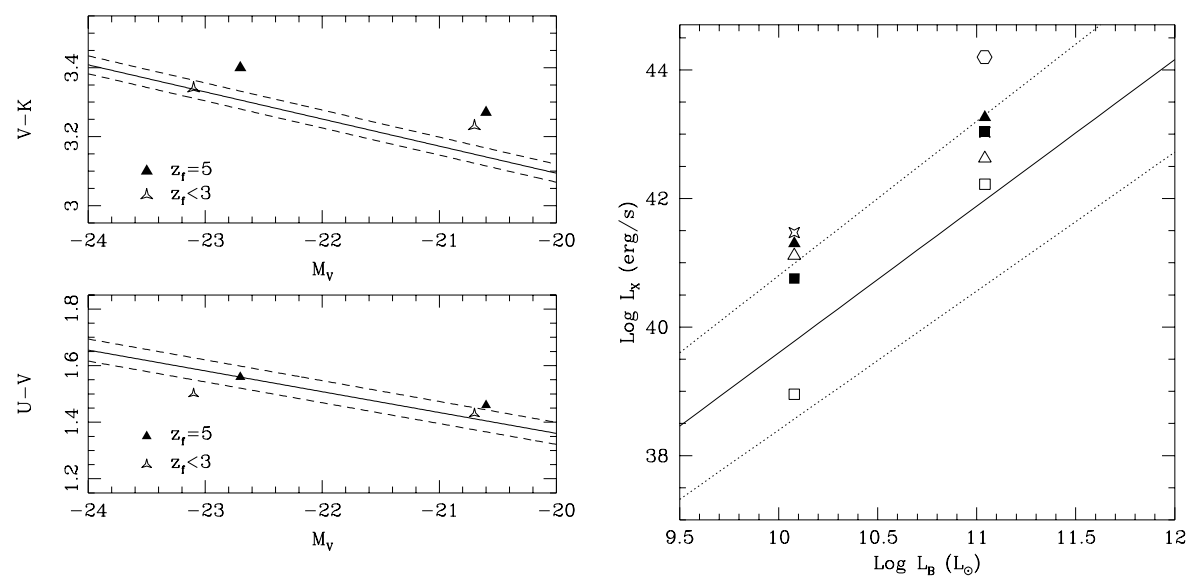

Figure 1: Left: Predicted Color-Magnitude relation for our models and different redshift of formation compared with the fit to the Bower et al. (2002b) data (solid line). A measure of the observational scatter is given by dashed lines. Right: Prediction for the $L_{B}-L_{X}$ relation for our model galaxies compared to the best fit of O'Sullivan et al.'s (2001, solid line) data, excluding bright cluster and group ellipticals as well as galaxies hosting an AGN. The dotted lines give a measure of the scatter. Filled triangles: La1, Ha1. Open squares: L0, H0. Open triangles: La1, Ha1 in which we fix $[\mathrm{Fe} / \mathrm{H}]=0$. Stars: Models $\mathrm{L}$ and $\mathrm{H}$ with $M_{\mathrm{acc}}=0.007 M_{\text {lum }}$. Filled squares: Models L and H with $M_{\text {acc }}=0.005 M_{\text {lum }}$. Open hexagon: Ha1 with R=6R $R_{\text {eff }}$.

The work was supported by Australian Research Council under the Linkage International Award and Discovery Project schemes and by MIUR under COFIN03 prot. 2003028039.

\section{References}

[] Arimoto, N., Matsushita, K., Ishimaru, Y., Ohashi, T., Renzini, A. 1997, ApJ, 477, 128

[] Bower, R.G., Lucey, J.R., Ellis, R.S. 1992a, MNRAS, 254, 589

[] Ciotti, L., D’Ercole, A., Pellegrini, S., Renzini, A. 1991, ApJ, 376, 380

[] Kawata, D., Gibson, B.K. 2003a, MNRAS, 340, 908

[] Kawata, D., Gibson, B.K. 2003b, MNRAS, 346, 135

[] Mathews, W.G., Brighenti, F. 2003, ARA\&A, 41, 191

[] Matsumoto, H., Koyama, K., Awaki, H., Tsuru, T., Lowenstein, M., Matsushita K. 1997, ApJ, 482, 133

[] O'Sullivan, E., Forbes, D.A., Ponman, T.J. 2001, MNRAS, 328, 461

[] Pipino, A., Matteucci, F. 2004, MNRAS, 347, 968 (PM04)

[] Pipino, A., Kawata, D., Gibson, B.K., Matteucci, F. 2005, A\&A submitted

[] Pipino, A., Matteucci, F., Borgani, S., Biviano, A. 2002, NewA, 7, 227 (P02)

[] Tozzi, P., Rosati, P., Ettori, S., Borgani, S., Mainieri, V., Norman, C. 2003, ApJ, 593, 705 